(2) Open Access Full Text Article

\title{
Low high-density lipoprotein cholesterol: current status and future strategies for management
}

\author{
This article was published in the following Dove Press journal: \\ Vascular Health and Risk Management \\ 28 October 2010 \\ Number of times this article has been viewed
}

\author{
Vibhuti Singh' \\ Rakesh Sharma \\ Ajoy Kumar ${ }^{3}$ \\ Prakash Deedwania ${ }^{4}$ \\ 'University of South Florida \\ College of Medicine, and Suncoast \\ Cardiovascular Center, St. Petersburg, \\ Florida, USA; ${ }^{2}$ Heart and Vascular \\ Institute of South Arkansas, University \\ of Arkansas for Medical Science, Little \\ Rock, Arkansas, USA; ${ }^{3}$ Family Practice \\ Training Program, Bayfront Medical \\ Center, St. Petersburg, Florida, USA; \\ ${ }^{4}$ Cardiology Division, VACCHCS \\ Fresno, CA, UCSF School of Medicine, \\ San Francisco, CA, USA
}

\begin{abstract}
Atherosclerotic cardiovascular disease is the foremost cause of death and disability in the Western world, and it is rapidly becoming so in the developing nations. Even though the use of statin therapy aiming at the low-density lipoprotein cholesterol (LDL) has significantly reduced cardiovascular events and mortality, substantial residual cardiac events still occur in those being treated to the currently recommended targets. In fact, residual risk is also seen in those who are treated "aggressively" such as the "high risk" patients so defined by the National Cholesterol Education Program (NCEP) Adult Treatment Panel III (ATP III). Consequently, one must look for the predictors of risk beyond LDL reduction. High-density lipoprotein cholesterol (HDL) is the next frontier. The protectiveness of elevated HDL against atherosclerosis is well described in the literature. HDL subdues several atherogenic processes, such as oxidation, inflammation, cell proliferation and thrombosis. It also helps mobilize the excess LDL via reverse cholesterol transport. Low levels of HDL have been shown to be independent predictors of risk. Thus, therapies to raise the HDL hold promise for additional cardiac risk reduction. In this regard, several randomized trials have recently tested this hypothesis, especially in patients at high risk. In addition to the use of aggressive lifestyle modification, clinical outcomes have been measured following augmentation of HDL levels with various treatment modalities, including aggressive statin therapy, combination therapy with fibrates and niacin, and direct HDL-raising drug treatments. These data for low HDL as an independent risk factor and as the new treatment target are reviewed in this paper.

Keywords: HDL, hypoalphalipoproteinemia, low HDL cholesterol, HDL, atherosclerosis risk reduction, statins, CETP inhibitors, niacin, dyslipidemia, reverse cholesterol transport, liver X-receptor agonists, PPAR-alpha agonists, Cordaptive ${ }^{\mathrm{TM}}$, D-4F agonists, farnesoid X-receptor antagonists, apolipoprotein-A agonists, fibrates
\end{abstract}

\section{Introduction}

Atherosclerotic heart disease continues to be the foremost cause of mortality in the United States for both men and women. ${ }^{1,2}$ It is well established that statins significantly reduce the number of recurrent cardiovascular events and have been the principal contributor for reduction in coronary heart diseases (CHD) death rates over the past 2 decades. However, the management of dyslipidemia in both the primary and secondary prevention settings has focused on low-density lipoprotein cholesterol (LDL) reduction as the primary target. Nonetheless, a worrisome number of residual events still occur in patients who are on adequate statin treatment as seen in several of the major randomized trials. For example, the Pravastatin or Atorvastatin Evaluation and Infection Therapy- Thrombolysis in Myocardial Infarction 22 (PROVE IT-TIMI 22), the Treating
Correspondence: Vibhuti Singh USF College of Medicine, Suncoast Cardiovascular Center, 60I-7th Street South, St. Petersburg, FL 3370I USA Email vsingh@health.usf.edu or vnsingh@ post.harvard.edu 
to New Targets (TNT), and the Incremental Decrease in End Points Through Aggressive Lipid Lowering (IDEAL) trials have now demonstrated that even when LDL was adequately lowered with statin therapy, a substantial number of residual cardiovascular events continued to occur. ${ }^{3-5} \mathrm{~A}$ treatment strategy solely targeting the LDL with statins, therefore, may not be the best lipid treatment strategy for CHD management. Therapeutic lipid treatment strategies that involve additional targets beyond mere LDL reduction must be explored.

High-density lipoprotein cholesterol (HDL) is one such lipid molecule that bears an inverse relationship with CHD and incidence of recurrent cardiovascular events. It has a cardio-protective effect, and higher levels of HDL may impart longevity. Data from the Framingham Heart Study have shown that subjects with the highest HDL levels exhibit the lowest risk of developing CHD. Prospective, observational studies conducted throughout the world have consistently demonstrated that high serum levels of HDL are associated with reduced risk for CHD development and related complications such as myocardial infarction (MI), stroke, and death, whereas low serum levels of this lipoprotein are correlated with increased risk for cardiovascular morbidity and mortality in both men and women. Low serum levels of HDL also portend increased risk for cardiovascular morbidity and mortality in patients treated with drug-eluting stents.

HDL and its sub-fractions are able to provide cardiac protection through various anti-atherogenic actions, such as their effectiveness against inflammation, oxidation, thrombosis, and cell proliferation. Among lipoproteins, HDL is also unique, in that, unlike atherogenic lipoproteins, which infiltrate arterial walls and deposit cholesterol and lipid within resident macrophages in the subendothelial space, HDL promotes the mobilization and clearance of excess lipid away from the arteries in a series of reactions collectively termed the reverse cholesterol transport. Numerous therapeutic agents are being developed in an attempt to modulate serum levels of HDL as well as its functions. Thus, a new therapeutic strategy seems to be taking shape. HDL is fast becoming the next major target to be tackled in the field of lipidology, in which raising the level of HDL is being considered highly desirable. In recent years both basic science and clinical research studies have provided data suggesting that therapies that raise HDL may augment risk reduction in patients with CHD.

This review will focus on the trials and tribulations of the mechanisms, magnitude of risk, and the methods of raising HDL for further reduction of cardiovascular risk beyond LDL by raising HDL levels or modifying its properties.

\section{Low HDL (hypoalphalipoproteinemia)}

Low levels of HDL, also known as hypoalphalipoproteinemia (HA), includes a variety of conditions, ranging from mild to severe, in which plasma concentrations of alpha lipoproteins or HDL are below the NCEP cutoff for desirable HDL level described above. The etiology of HDL deficiency range from secondary causes, such as smoking, to primary or familial causes including specific genetic mutations, such as Tangier disease and fish-eye disease. Hypoalphalipoproteinemia has no clear-cut definition. An arbitrary cutoff used frequently in the old literature includes a HDL level that falls within the 10th percentile of HDL levels.

A more practical definition derives from the theoretical cardioprotective role of HDL. The US National Cholesterol Education Program (NCEP) Adult Treatment Panel III (ATP III) redefined the HDL level that constitutes a formal CHD risk factor. The minimum desirable level was raised from 35 to $40 \mathrm{mg} / \mathrm{dL}$ for both men and women. For persons with metabolic syndrome, however, the designated HDL levels that contribute to the syndrome are gender-specific. For men, a desirable HDL level is still one that is above $40 \mathrm{mg} / \mathrm{dL}$, but for women, the desirable level is greater than $50 \mathrm{mg} / \mathrm{dL}$ to satisfy the definition of metabolic syndrome.

\section{HDL: an independent CHD risk factor}

In the latest report, the NCEP ATP-III guidelines clearly indicate that low HDL is a significant and independent risk factor for $\mathrm{CHD} .{ }^{6}$ The relationship is inversely proportional, such that $1 \mathrm{mg} / \mathrm{dL}$ rise in HDL imparts $2 \%$ to $3 \%$ reduction in CHD risk. ${ }^{7}$ Low HDL is frequently associated with high triglycerides and remnant lipoprotein levels. Furthermore, low HDL is quite prevalent in the US according to the third National Health and Nutrition Examination Survey (NHANES III). Approximately $35 \%$ of adult men have $\mathrm{HDL}<40 \mathrm{mg} / \mathrm{dL}$ and $39 \%$ of women have $\mathrm{HDL}<50 \mathrm{mg} / \mathrm{dL} .{ }^{8}$ Analysis of data from the Framingham study revealed that there was a significant increase in CHD events in patients who had low HDL at all levels of LDL. ${ }^{9}$ Furthermore, it has been described that the patient with desirable LDL level $(100 \mathrm{mg} / \mathrm{dL})$ in association with low HDL ( $25 \mathrm{mg} / \mathrm{dL}$ ) level would have a CHD risk equivalent to a patient with elevated LDL $(220 \mathrm{mg} / \mathrm{dL})$ but a higher, desirable HDL level $(45 \mathrm{mg} / \mathrm{dL}){ }^{9}$ Thus, it has now become quite apparent that low concentration of HDL is a significant and independent risk predictor for CHD.

A prospective meta-analysis of 14 randomized trials was performed by the Cholesterol Treatment Trialists' Collaboration that included 90,056 subjects, including $21 \%$ 
with adult-onset (type 2) diabetes. After a mean follow-up of 5 years, with 8,186 deaths and 14,348 major cardiovascular events, the statin-treated patients with low HDL ( $\leq 35 \mathrm{mg} / \mathrm{dL})$ showed a significantly higher rate of major cardiovascular events than those with higher HDL levels $(>42.5 \mathrm{mg} / \mathrm{dL}) .{ }^{10}$ Thus, statin treatment alone does not appear to be good enough to eliminate the risk associated with low HDL. This analysis, again, suggests that a focus exclusively on LDL reduction falls short of best possible CHD risk reduction.

The European Prospective Investigation into Cancer and nutrition - Norfolk cohort (EPIC-Norfolk) and the Incremental Decrease in End Points through Aggressive Lipid Lowering (IDEAL) trials examined the relationship between plasma HDL level, HDL particle size, level of apolipoprotein (apo A-1), and their relationship with CHD events. ${ }^{11}$ In both trials, HDL was found to have a significant protective effect on recurrent $\mathrm{CHD}$ events. For apo A-1 and apo B, there was a protective/inverse relationship with risk for a major CHD event when HDL was over $70 \mathrm{mg} / \mathrm{dL}$ in the IDEAL study. The apo A-1 remained strongly inversely associated with CHD events in IDEAL, and HDL particle size and apo B in EPIC-Norfolk at any level of HDL. Similarly, the Treating to New Targets (TNT) study (subgroup analysis of 2661 subjects) showed that patients in the aggressively treated group who had achieved the lowest LDL levels of about $58 \mathrm{mg} / \mathrm{dL}$ still exhibited a significant risk of major cardiovascular events over the 5 year follow up if their HDL-C was low. ${ }^{12}$ The subjects in the highest HDL quintile (HDL $\geq 55 \mathrm{mg} / \mathrm{dL}$ ) showed significantly lower major CHD events than those in the lowest quintile (HDL $<37 \mathrm{mg} / \mathrm{dL}$, $P=0.03$; Q5 hazard ratio $[\mathrm{HR}]=0.61) .{ }^{11}$ Thus, even the most aggressive statin therapy to achieve very low levels of LDL does not eliminate the risk of CVD events when the HDL-C is low.

\section{HDL: production, pathophysiology and metabolism}

Lipid molecules, such as HDL and LDL, circulate in the plasma complex with proteins termed lipoproteins, which act as carriers. Such complexed plasma lipoproteins are macromolecular structures that are classified based on their physical properties, ie, density and electrophoretic mobility. The overall structure of all lipoproteins is similar. The nonpolar lipid molecules, ie, cholesteryl ester, and triglycerides (TGs), circulate in a core surrounded by more polar components, eg, free cholesterol, phospholipids, and proteins. The protein components, appropriately termed apolipoproteins, play a key role in lipoprotein metabolism. The major apolipoproteins of
HDL are alpha lipoproteins (ie, apolipoprotein A-1 apo A-1, apo A-2, and apo A-4). Alpha lipoproteins are soluble and can move between different classes of lipoproteins relatively freely. In contrast, the beta lipoproteins, also called apolipoprotein B (apo B), do not move as freely, and are mostly associated with lower-density lipoproteins. For example, apo B-100 constitutes LDL, very low-density lipoproteins (VLDL), VLDL remnants, and intermediate-density lipoproteins (IDL), while apo B-450 is associated with chylomicrons and the remnants.

HDL plays an important role in reverse cholesterol transport (RCT), extracting cholesterol from the peripheral tissues and routing it to the liver. In the general population, lower-than-normal HDL-C levels are inversely correlated with CHD; the risk of a coronary event is thought to increase $2 \%$ for every $1 \%$ decrease in HDL. However, extreme HDL deficiencies caused by rare autosomal recessive disorders, including familial HA, familial lecithin-cholesterol acetyltransferase (LCAT) deficiency, and Tangier disease, do not always correlate with more frequent CHD.

Apo A-1 is the major HDL protein, constituting approximately $70 \%$ of the apolipoprotein content of the HDL molecule. ${ }^{13}$ Apo A-1 is produced in the liver and intestine and is initially secreted as a lipid-void apo A-1 molecule, sometimes termed as pre-HDL particle, and the lipid component is subsequently added to it in the presence of transport proteins. One such cellular lipid transport protein, the adenosine triphosphate (ATP)-binding cassette transporter A-1 (ABCA-1), is required for normal lipidation of lipid-void apo A-I lipoprotein. During this process of lipidation phospholipids and unesterified cholesterol elements are effluxed and added to the lipid-void apoA-1 molecules. When ABCA-1 is absent or dysfunctional, the apo A-1 gets catabolized too quickly leading to lower levels of apo A-1, and thus lower levels of HDL. Interestingly, the ABCA-1 transporter protein is expressed in almost all tissues; however, only liver and intestine are the primary sites where lipidation of lipid-void apo A-1 takes place.

\section{Components of plasma HDL}

Plasma HDL is a small, dense, spherical lipid-protein structure, with the lipid and protein components each constituting half of the molecule. Major lipid constituents include phospholipid, cholesterol, cholesteryl esters, and triglycerides, while the main proteins constituents include apo A-1 (molecular weight, 28,000 Da) and apo A-2 (molecular weight, 17,000 Da). Other minor proteins found in smaller amounts include apo C-1, apo C-2, apo C-3, and apo-E. 
HDL molecules vary in molecular content and are relatively heterogeneous. Based on size and density, the HDL can be classified into larger, less dense HDL-2 and smaller, denser HDL-3 particles. The majority of the HDL exists as HDL-3. However, individual variability in HDL levels is usually due to variability in the quantity of HDL-2 particles.

\section{Properties of HDL}

\section{Antiatherogenesis: RCT}

RCT is considered to be the principal mechanism through which HDL exerts its cardioprotective effect. Macrophages present in the arterial wall are primarily involved in this process. Acting as cellular scavengers, the macrophages absorb products of wear and tear, such as dead cells, modified lipoproteins, and extracellular debris. A large volume of cholesterol is phagocytosed in this process, too. Cholesterol, in its unesterified form, is toxic to macrophages and can ultimately lead to apoptosis. Such apoptosis, however, is avoided, and atherosclerosis begins to take a foothold in the presence of an esterifying enzyme, acyl-coenzyme-A cholesterol O-acyltransferase-1 (ACAT-1). The process of atherosclerosis begins as follows. First, the ACAT-1 esterifies the cholesterol molecule making it hydrophobic, which, in turn, avoids going through cell death or apoptosis and begins to accumulate as lipid droplets within the cytoplasm. Cells loaded with such lipid droplets are called foam cells. The HDL molecule gets involved at this very step to prevent the formation of foam cells (anti-atherogenic effect). It intercepts the cholesterol molecules that efflux from the macrophages and undergoes lipidation. Several macrophage receptors are required for actively effluxing the cholesterol from the macrophages before lipidation of HDL. These receptors include ATPbinding cassette transporter A-1 (ABCA-1), ATP-binding cassette transporter G-1 (ABCG-1), and scavenger receptor class-B type-1 (SR-B1. The ABCA-1 receptor molecule is responsible for lipidating only newly secreted apoA-1, while the ABCG-1 and SR-B1 receptors can directly lipidate HDL molecule. The ABCA-1 and ABCG-1 receptors are regulated by the nuclear liver-X-receptors-alpha (LXR-alpha) and LXR-beta.

Thus, the first step of RCT involves efflux of cholesterol from the macrophages before it could get esterified by ACAT-1 within the macrophage. The efflux occurs under the influence of LXR-alpa and LXR-beta that regulates the macrophage receptors ABCA-1, ABCG-1 and SR B-1, and facilitate lipidation of apo A-1 and HDL. Once cholesterol gets associated with HDL, it becomes esterified through the action of another enzyme, LCAT. ${ }^{13}$ Cholesterol ester, thus produced, is, again, hydrophobic and it accumulates to the center of the lipoprotein molecule, producing mature HDL. ${ }^{14}$ Subsequently, the mature HDL is transported to the liver where it releases the cholesterol for excretion into the bile. Selective uptake of HDL by the liver is mediated by the hepatic receptor, scavenger receptor class $B$ type 1 (SR-B1). Once in the liver, the cholesterol is either converted to bile acids before excretion or gets excreted directly into the bile.

In the absence of LCAT, mature HDL particles fail to form, and, thus, the immature HDL molecules become dysfunctional, and remain incapable of carrying out the RCT process. Even heterozygous patients for LCAT gene have been shown to be unable to form mature HDL molecules, and exhibit evidence of progressive atherosclerosis as indicated by increased carotid intima-media thickness (CIMT).

There is an alternative pathway that utilizes cholesterol ester transfer protein (CETP). CETP mediates the transfer of esterified cholesterol from HDL to apo B-containing proteins (eg, apo B-100) in equal 1:1 exchange for TG. Increased CETP activity lowers HDL and may reduce the amount of cholesterol delivered back to the liver via direct RCT. As a result pro-atherogenic activity may become enhanced. Suppression of CETP activity has recently been the focus of development of new therapeutic agents for raising plasma levels of HDL. ${ }^{15}$

\section{Additional beneficial properties of HDL}

Several other properties of HDL beyond its involvement in the RCT pathway exert additional anti-atherogenic influence. ${ }^{16,17}$ HDL has been shown to directly stimulate tissue plasminogen activator (tPA) and inhibit the expression of plasminogen activator inhibitor-1 (PAI-1). It also decreases the expression of adhesion molecules, such as vascular cell adhesion molecule-1 (VCAM-1), intercellular adhesion molecule-1 (ICAM-1) and monocyte chemoattract protein-1 (MCP-1). This action is accomplished by suppression of sphingosine kinase causing reduced production sphingosine-1-phosphate and subsequently, lowering the quantity of nuclear factor kappa-B (NK-kappa-B) which is required for adequate expression of the adhesion molecules.

In addition, HDL promotes vasodilation by enhancing the formation of prostacyclin by acting as a substrate (ie, arachidonic acid) which is acted upon by the enzyme, cyclooxygenase, present on the endothelial cells. HDL has been implicated in curtailing the apoptosis and promoting the proliferation and migration of endothelial cells especially when damaged through the inhibition of the activation of 
caspase-3 and caspase-9. Finally, HDL has been shown to directly enhance nitric oxide production by stabilizing $m$ RNA transcripts for nitric oxide synthase in the vascular endothelium. Vascular dilatation and myocardial perfusion both have been shown to vary in proportion to the HDL levels.

Because of the above properties, the propensity and risk for thrombosis are significantly reduced in the presence of higher levels of HDL. By increasing the endothelial production of tPA, prostacyclin, and nitric oxide, HDL reduces the platelet reactivity and coagulability. It also decreases the production of thromboxane A2 in the platelets. Generation of thrombin is reduced as does the thrombin-dependent platelet aggregation. HDL also enhances protein-C and protein-S activation, and reduces formation of the tissue factor curtailing the coagulability.

\section{Variants, prevalence and causes of low HDL}

\section{Variant apolipoproteins}

The variant apo A-I Milano appears to share HDL properties. Low levels of apo-A-1 Milano are associated with increased risk of atherosclerotic cardiovascular disease, and increasing its level has been shown to be protective. Other less well-known variants that include apo A-1 Marburg, apo A-1 Giessen, apo A-1 Munster, and apo A-1 Paris, cause hypoalphalipoproteinemia, but do not seem to increase the risk of atherosclerosis.

\section{Prevalence of HA (low HDL)}

Hypoalphalipoproteinemia is frequently found in patients with CHD. Research indicates that $58 \%$ of patients with CHD have HDL levels below the 10th percentile of normal values. On the other hand, according to the NHANES III survey report, approximately $35 \%$ of adult men have $\mathrm{HDL}<40 \mathrm{mg} / \mathrm{dL}$ and $39 \%$ of women have HDL $<50 \mathrm{mg} / \mathrm{dL}$ in the US. Prevalence of low HDL is higher in certain ethnic populations, such as those of South Asian Indian descent.

\section{Causes of low HDL/HA}

HA may be caused by familial or primary and secondary disorders that are associated with low plasma levels of HDL. A number of various primary (or familial) causes as well as secondary causes and associations are listed below:

\section{Familial or primary causes}

Decreased or absent synthesis of apo A-I due to a defect at the level of the genes is the cause of hypoalphalipoproteinemias characterized by apo A-1/apo C-3 and apo A-1/apo
C-3/apo A-4 deficiency. However, the etiology of the low levels of HDL is unclear for most of the remaining familial HAs. Some of the other underlying causes include increased catabolism, decreased synthesis, and altered equilibration of HDL between intravascular and extravascular spaces. Whatever the cause, these disorders are associated with altered HDL composition as well as altered equilibration of cholesterol, among the various lipoprotein classes. Main familial alphalipoproteinemias include familial apo A-1 deficiency and structural mutations, familial LCAT deficiency, Tangier disease, and several less defined familial conditions such as familial HDL deficiency, familial apo A-1 and apo C-3 deficiency (apo A-1 absence), familial deficiency of apo A-1 and apo C-3, fish-eye disease (partial LCAT deficiency), familial HA, and presence of Apo A-1 variants, eg, apo A-1 Milano, apo A-1 Marburg, apo A-1 Giessen, and apo A-1 Munster. ${ }^{17}$

\section{Secondary causes}

Secondary causes or conditions associated with hypoalphalipoproteinemia include obesity, metabolic syndrome, physical inactivity, type 2 diabetes, cigarette smoking, end-stage renal disease, hypertriglyceridemia, treatment with probucol, androgens or progestins, use of high-dose thiazide diuretics and high-dose beta blockers, very low-fat diet, dysglobulinemia, severe liver disease, malabsorption, malnutrition, and severe inflammatory disease. ${ }^{17}$

\section{Special populations: South Asian Indians}

Americans of South Asian descent, especially those from the Indian subcontinent, African Americans, and Hispanics represent a special group of people that show a much greater prevalence of cardiovascular disease and coronary risk factors as compared with white American counterparts. Persons who can claim their origin to the Asian and Pacific Islander population consist of two very diverse kinds of population with regards to their overall cardiovascular disease risk. South Asia is generally represented by India, Pakistan, Bangladesh, Nepal, and Sri Lanka; and South-East and Far East Asia is represented by Singapore, Malaysia, Thailand, the Philippines, China, Hong Kong, and Japan. The South Asian Indian Americans form a high risk subset and exhibit a 2-fold higher cardiac mortality rate compared with Caucasians, irrespective of their gender, religion, social class, or dietary practices. ${ }^{18}$ By contrast, persons of East Asian and Far East descent, such as those of Chinese and Japanese origin, experience a much lower burden of cardiovascular prevalence and concomitant mortality rates. 
The explanation for such a high prevalence of CHD and mortality rates in the South Asian Indians appears to be a combination of genetic predisposition and environmental interaction. Increased prevalence of low HDL has been found to be one such underlying factor. An analysis of the INTERHEART study highlights the high prevalence of low plasma levels of HDL in South Asian persons as compared with other Asian counterparts. ${ }^{19}$ In this study, over $80 \%$ of participants, both with and without the history of prior acute myocardial infarction (AMI), exhibited low levels of HDL, ie, less than $50 \mathrm{mg} / \mathrm{dL}$ in women and under $40 \mathrm{mg} / \mathrm{dL}$ in men. The INTERHEART study, conducted in 52 countries at about 262 study sites around the World, evaluated 15,152 patients with their first occurrence of AMI and 14,820 age- and gender-matched controls. Focusing on South Asians, roughly a third of the participants (5,731 cases of AMI and 6,459 controls) were subjected to a sub-analysis comparing lipid and lipoprotein levels for various Asian sub-populations and non-Asians. The prevalence of low HDL levels was much higher in the South Asian population ( $82 \%$ of AMI cases and $81 \%$ of controls) than in the other (60\% of AMI cases and $50 \%$ of controls). Furthermore, while the presence of higher levels of HDL correlated with a decreased risk of AMI, the extent of decrease in risk seemed to be less prominent for South Asians than for other Asians (odds ratio for 1 SD elevation in HDL 0.87 in South Asians vs 0.77 in others. Thus, while a 1 SD increase in HDL was associated with a $23 \%$ reduction in AMI risk in other Asians, it caused a mere a $13 \%$ risk reduction in the South Asians. Increases in apo A-1, however, seemed to be equally protective. The lower levels of HDL in South Asians observed in the INTERHEART study reinforce the relevance of interventions aimed at raising HDL in this population. The authors concluded: 'The low level of HDL among South Asians requires further study and targeted intervention'.

A study reported by Dodani et al attempted to identify apo A-1 polymorphisms, the main protein component of HDL and explore its association with low HDL levels in South Asians. ${ }^{20}$ The study was conducted on 30 South Asians. DNA extraction, and sequencing of apo A-1 gene were performed. DNA sequencing revealed 6 novel apo A-1 single nucleotide polymorphisms (SNPs) in South Asians, one of which (rs35293760, C938T) was significantly associated with low $(<40 \mathrm{mg} / \mathrm{dL})$ HDL levels $(P=0.004)$. The association was also seen with total cholesterol $(P=0.026)$ and LDL levels $(P=0.032)$. This pilot work has highlighted some of the gene-environment associations that could be responsible for low HDL and, possibly the excess burden of CHD among South Asians.

Further larger studies are required to explore and further elucidate these associations which may, one day, lead to better targeted therapies.

\section{Management of low HDL}

The NCEP ATP III guidelines now recognize low HDL level as a categorical and independent risk factor for CHD. Low serum HDL level is also one of the defining features of metabolic syndrome. It is estimated that approximately $35 \%$ of men and 39\% of women residing in the United States have low serum levels of HDL. This is an important and widely prevalent risk factor that must be addressed in the clinical contexts of primordial, primary, and secondary prevention.

Attempts to raise HDL are becoming increasingly frequent in the literature and most agree that raising HDL should be one of the goals in the management of atherogenic dyslipidemias for CHD risk reduction. While statin, niacin, and fibrate therapies have been demonstrated to increase HDL to a varying extent and improve cardiovascular outcomes, a number of newer therapeutic agents have now appeared on the horizon that hold promise for raising HDL. These agents include infusible HDL type agents, new peroxisome proliferator activated receptor-alpha (PPAR-alpha) agonists, liver-X receptor-alpha (LXR- $\alpha$ ) agonists, CETP inhibitors, D-4F and other apo A agonists, Cordaptive ${ }^{\mathrm{TM}}$, and farnesoid $\mathrm{X}$ receptor (FXR) antagonists. Each of these novel therapies will be briefly reviewed in the following sections.

\section{International and national guidelines and low HDL American Heart Association (AHA) \\ Guidelines for women}

In light of the increasing prevalence of CHD in women, AHA highlighted the evidence based guidelines for CHD prevention in women in its 2004 guidelines as well as its update in 2007.21,22 The AHA guidelines recommend lipid goals of LDL under $100 \mathrm{mg} / \mathrm{dL}$, HDL to be above $50 \mathrm{mg} / \mathrm{dL}$, triglycerides $<150 \mathrm{mg} / \mathrm{dL}$, and non-HDL $<130 \mathrm{mg} / \mathrm{dL}$. Therapeutic lifestyle changes were recommended as the main therapy. However, for women with persistent low HDL the AHA guidelines state that niacin or fibrate therapy should be initiated. ${ }^{22}$

\section{NCEP ATP III statement}

The NCEP ATP III guidelines state that LDL is the primary target for lipid-lowering therapy. In their 2004 update, however, they recognized that elevated triglyceride and low HDL levels contribute to CHD risk, even after LDL goals have been met. 
Thus, combination therapy may be required in this regard. The 2004 update to the NCEP ATP III guidelines has the following additional recommendation: 'For those high-risk patients who have elevated triglycerides or low HDL levels, addition of a fibrate or nicotinic acid to the routine LDLlowering therapy can be considered'. ${ }^{23}$

\section{American Diabetes Association (ADA) guidelines}

It is well known that the patients with type 2 diabetes have a 2- to 4-fold higher risk for CHD. The most common form of dyslipidemia in the diabetics includes co-existence of high levels of TG and low serum levels of HDL. ${ }^{24}$ ADA 2008 guidelines recommend initiation of statin therapy to lifestyle therapy irrespective of the lipid levels for all diabetics who have either manifest CHD or $\geq 1$ other CHD risk factor and are over the age 40 in the absence of manifest CHD. For lower-risk diabetics, such as those without diagnosed CHD and those younger than 40 years, addition of statin should be considered if LDL continues to exceed $100 \mathrm{mg} / \mathrm{dL} .^{25}$ In addition, the guidelines recommend attainment of TG levels below $150 \mathrm{mg} / \mathrm{dL}$ and HDL above $40 \mathrm{mg} / \mathrm{dL}$ in men and $50 \mathrm{mg} / \mathrm{dL}$ in women.

According to the guidelines, combination therapy with statin and an additional agent such as a fibrate or niacin may be efficacious for treatment of these mixed lipid abnormalities (ie, high LDL, low HDL, and elevated TG). Despite the concern over niacin use and potential for the interference with glucose control, it has been shown that niacin in moderate doses, ie, 750 to $2000 \mathrm{mg}$ daily, significantly improves LDL, HDL, and TG levels and causes only minor variation in glucose level that can be generally corrected through therapeutic adjustment.

\section{American College of Cardiology (ACC) and ADA: combined recommendations}

ACC and ADA published a consensus statement in 2008 that provides recommendations for patients with elevated global cardiometabolic risk (CMR). Patients with elevated CMR often present with mixed type of atherogenic dyslipidemia characterized by elevated TG, low HDL, and increased numbers of small, dense LDL particles. ${ }^{26}$ Other than dyslipidemia, such patients also tend to have central obesity, insulin resistance, and hypertension. The consensus statement recommends LDL and non-HDL goals for patients with CMR and lipoprotein abnormalities based on their CHD risk. Statins are recommended as the first line of therapy; however, combination therapy is recommended for patients on statin therapy who also have low HDL or elevated non-HDL, especially if apo B levels are also high. According to the guidelines, niacin is the preferred agent for use in combination with statins because of the availability of greater evidence for CHD event reduction with niacin compared with fibrates. ${ }^{26}$

\section{General management of low HDL Diagnosis of HA}

Most individuals are diagnosed with HA based on the results of a routine lipid profile measurement. This finding of a HDL-C level can be useful as an independent factor in assessing coronary artery disease (CAD) risk and further management. The Guidelines in the ATP III report emphasized the importance of HDL-C; the level of HDL was considered to be a significant risk factor, and the "desirable" level was changed from $35 \mathrm{mg} / \mathrm{dL}$ to $40 \mathrm{mg} / \mathrm{dL}$ for both men and women. Interestingly, to satisfy the definition of metabolic syndrome, the cutoff risk level is stated to be $40 \mathrm{mg}$ / $\mathrm{dL}$ for men, but for women it is $50 \mathrm{mg} / \mathrm{dL}$.

\section{Principles and goals of HDL therapy}

The basic purpose of the management of low HDL and related dyslipidemias is to reduce the risk of atherosclerosis and its sequelae. For HDL, the ATP III report states 'Low HDL cholesterol is a strong independent predictor of CHD'. In the present guidelines, presence of low HDL-C level both modifies the goal for LDL-lowering therapy and is used as a risk factor to estimate 10-year risk for CHD. While contemplating therapy, the ATP III recommends that the causes and associations of low HDL must be ascertained and treated to their target as the first step before considering specific therapy. These causes include type II diabetes, overweight status and obesity, elevated TGs, lack of physical activity, associated insulin resistance, cigarette smoking, very high carbohydrate intake ( $>60 \%$ of calories), and use of certain pharmacologic agents such as progestational drugs, and anabolic steroids.

Unlike the establishment of a target LDL level, The ATP III report does not provide a specific level which a low HDL concentration should be raised to. The panel stated that although clinical trial results suggest that raising HDL may reduce CHD risk, the evidence is yet insufficient to specify a target level of therapy. It is also influenced by the fact that currently available drugs do not robustly raise HDL. At the present juncture, the low HDL levels should be managed in the following manner: First, reduction of LDL to defined target levels remains as the primary goal. Metabolic syndrome is the second target after the LDL goal has been achieved. The emphasis should then shift to therapeutic lifestyle changes, such as weight reduction and 
increased physical activity when the metabolic syndrome is present. Diagnosis of metabolic syndrome can be made in the presence of 3 or more of the following 5 risk factors: (a) abdominal obesity, with a waist circumference over 40 inches $(100 \mathrm{~cm})$ (in men) or above 35 inches $(88 \mathrm{~cm})$ (in women); for South Asians the cutoff is 35 inches $(88 \mathrm{~cm}$ ) (for men) and 32 inches ( $80 \mathrm{~cm}$ ) (for women) respectively, (b) TG level of $150 \mathrm{mg} / \mathrm{dL}$ or greater, (c) HDL level below $40 \mathrm{mg} / \mathrm{dL}$ (males) or less than $50 \mathrm{mg} / \mathrm{dL}$ (females), (d) blood pressure at or above $130 \mathrm{mmHg}$ systolic and greater than or equal to $85 \mathrm{mmHg}$ diastolic, and (e) fasting glucose levels at or above $110 \mathrm{mg} / \mathrm{dL}$.

The interaction between low HDL and hypertriglyceridemia requires special attention because such mixed dyslipidemia is associated with aggressive atherogenecity. The ATP III reported that when a low HDL level is present in association with high levels of TG (200 to $499 \mathrm{mg} / \mathrm{dL}$ ), secondary priority, right after achievement of LDL goals, must go to achieving the non-HDL goal. These target levels as defined by NCEP ATP-III are as follows:

(i) In the patients with established CHD or a CHD risk equivalent (10-year risk for CHD $>20 \%$ ), the LDL goal is $<100 \mathrm{mg} / \mathrm{dL}$, and the goal for non-HDL choleseterol (LDL plus VLDL) is $<130 \mathrm{mg} / \mathrm{dL}$;

(ii) In persons with multiple (2+) risk factors and 10-year risk of equal to or less than $20 \%$, the LDL goal is $<130$ $\mathrm{mg} / \mathrm{dL}$, while the non-HDL goal is $<160 \mathrm{mg} / \mathrm{dL}$; and

(iii) In persons with $0-1$ risk factor, the LDL goal is $<160 \mathrm{mg} / \mathrm{dL}$, and that for the non-HDL is $<190 \mathrm{mg} / \mathrm{dL}$.

\section{Treatment of isolated low HDL}

Treatment of isolated low HDL has gained increasing importance in the literature in the recent years. According to the ATP III, if a patient's TG levels are below $200 \mathrm{mg} / \mathrm{dL}$ and the HDL is low (isolated low HDL), the administration of drugs that increase HDL (fibrates or nicotinic acid) can be considered. Statins exert only a modest effect. Treatment for isolated low HDL is provided mainly in the patients who have manifest CHD or CHD risk equivalents. Current recommendations include:

(a) Identification of patients whose diet is very low in fat. A low HDL level in this setting is rarely associated with an increased risk for premature CHD;

(b) Identification and correction of secondary factors, such as smoking cessation, weight management, increased physical activity, elimination of medications that are known to lower HDL levels, and optimal diabetes control; (c) Use of pharmacologic agents to raise the HDL level in otherwise healthy persons. Though no published clinical trials are available yet they demonstrate a benefit. Nonetheless, individuals at high risk require further assessment for CHD risk, with an evaluation that includes a family history, measurements of apoprotein, lipoprotein $\left(\mathrm{Lp}_{(\mathrm{a})}\right)$, and electron beam CT scanning;

(i) Niacin is the most effective agent currently available. However, many patients with isolated HA do not respond well to niacin.

(ii) Gemfibrozil and fenofibrate modestly raise the HDL level. They are most effective in the setting of concomitant hypertriglyceridemia.

(iii) Alcohol tends to raise some HDL subfractions. However, no clinical trial data are available to demonstrate any positive role for raising HDL levels with alcohol in order to reduce cardiovascular events in patients with CHD.

\section{Therapeutic lifestyle changes}

\section{Dietary interventions}

Diets that are very low in fat are associated with low HDL levels too. However, increasing the fat content of a patient's diet for the purpose of raising HDL is not recommended. Dietary management should follow the NCEP guidelines for lowering frequently associated LDL cholesterol, which is the primary target in lipid management. The NCEP has recommended a therapeutic lifestyle-change diet, which should be incorporated in the treatment of all patients. The following are the recommendations:

(a) Patients should reduce their intake of saturated fats to less than $7 \%$ of their total calorie (energy) intake. Their cholesterol intake should be reduced to less than $200 \mathrm{mg} /$ day. Trans fatty acids (the HDL-lowering, LDL-raising fats) should be kept to a minimum. Polyunsaturated fats should constitute up to $10 \%$ of total energy intake, and monounsaturated fats, up to $20 \%$ of total energy intake. Total fat intake, therefore, should be in the range of $25 \%$ to $35 \%$ of total energy intake.

(b) Carbohydrates (complex carbohydrates from grains, whole grains, fruits, and vegetables) should constitute $50 \%$ to $60 \%$ of total energy intake.

(c) Patients should consume 20 to $30 \mathrm{~g}$ /day of fiber.

(d) The protein content should be approximately $15 \%$ of the total calorie intake.

(e) In order to maintain a desirable body weight and to prevent weight gain, the total amount of energy 
consumed must be balanced in terms of energy intake and expenditure.

\section{Exercise/physical activity}

Physical activity, especially regular physical activity, should be strongly encouraged in all persons, and particularly in those with sedentary habits and low HDL state. According to the NCEP guidelines, daily activity and energy expenditure should include at least moderate physical activity, with the daily energy expending approximating 840 kilo joules or greater every day.

\section{Raising HDL: trials (and tribulations)} CETP inhibitors

One of the pharmacologic approaches that have recently undergone clinical evaluation in the randomized trials includes attempts at elevation of HDL level through the inhibition of the enzyme, CETP. The CETP tends to negatively influence the reverse cholesterol transport pathway by mediating the stoichiometric 1:1 exchange of triglycerides for esterified cholesterol (cholesteryl ester) between apolipoprotein B100-containing lipoproteins and HDL molecules. As a result, it lowers HDL level and may reduce the amount of cholesterol delivered back to the liver via direct RCT. Increased CETP activity is pathologic, and tends to accelerate atherogenesis. Therefore, inhibition of CETP activity can potentially provide a therapeutic benefit for patients with CHD by allowing HDL particles to accumulate and be more available for augmenting the RCT process that scavenges cholesterol from the tissues. Several approaches to inhibit CETP activity and, thus, augment HDL level have been examined including use of anti-CETP antibodies, antisense oligonucleotides, and a vaccine that induces antibodies for the inhibition of CETP action.

Genetic mutations causing CETP deficiency result in increased levels of HDL. This information in the recent years led to intense interest in the development of CETP inhibitors as therapeutic agents for raising HDL. ${ }^{27,28}$ Individuals homozygous for the CETP mutation that leads to its dysfunction or deficiency, have been shown to be associated with elevations in HDL exceeding 100\%, along with more than $40 \%$ decrease in the level of LDL and apo-B as compared with familial controls. However, the CETP deficiency is not always predictably associated with decreased CHD risk. In fact, the studies evaluating the relationship between CETP inhibition and extent of atherosclerosis in animal models have shown mixed results. While studies with CETP transgenic mouse models show inconsistent effects on the progression of atherosclerosis, the studies using rabbit models of CETP inhibition consistently showed a reduction in atherosclerosis. For example, cholesterol-fed rabbits that were treated with the CETP inhibitor, torcetrapib, exhibited 4-fold increases in HDL associated with a $60 \%$ reduction in atherosclerosis.

Studies of the effects of torcetrapib on the vascular system (ILLUSTRATE, RADIANCE I and RADIANCE II trials)

One of the most illustrative studies, the Investigation of Lipid Level Management Using Coronary Ultrasound to Assess Reduction of Atherosclerosis by CETP Inhibition and HDL Elevation (ILLUSTRATE) trial, examined the effects of the addition of torcetrapib to atorvastatin therapy on the vascular system as assessed by intravascular ultrasound (IVUS). ${ }^{29} \mathrm{~A}$ total of 1188 patients with known CHD were treated with atorvastatin to bring their LDL levels below $100 \mathrm{mg} / \mathrm{dL}$ before randomization to either atorvastatin monotherapy or a combination therapy with atorvastatin and torcetrapib (60 mg). At the end of two years of follow-up, patients receiving torcetrapib showed a significant $61 \%$ increase in HDL, and a $20 \%$ reduction in LDL compared with those who received atorvastatin monotherapy. The primary end point of percent atheroma volume did not differ significantly between the torcetrapib/atorvastatin group and the atorvastatin group $(0.12 \%$ versus $0.19 \%, P=0.72)$. These patients, however, experienced a $4.6 \mathrm{mmHg}$ increase in blood pressure.

Another similar study, the Rating Atherosclerosis Disease Change by Imaging with a New CETP Inhibitor (RADIANCE 1), evaluated 850 patients with heterozygous familial hypercholesterolemia following the addition of torcetrapib to baseline atorvastatin therapy and measured the change in atherosclerosis by using CIMT as the main parameter. ${ }^{30}$ Patients in the torcetrapib/atorvastatin group had significantly higher HDL ( $82 \mathrm{mg} / \mathrm{dL})$ and lower LDL (115 mg/dL) compared with the atorvastatin monotherapy group $(52 \mathrm{mg} / \mathrm{dL}$ and $143 \mathrm{mg} / \mathrm{dL}$, respectively; $P<0.001$ for both comparisons). However, the increase in maximum CIMT was $+0.0047 \mathrm{~mm} /$ year in the torcetrapib/ atorvastatin group, which was not significantly different from the $+0.0053 \mathrm{~mm} /$ year increase observed in the atorvastatin monotherapy group $(P=0.87) .{ }^{30}$ Again, an average increase of $2.8 \mathrm{mmHg}$ in systolic blood pressure was observed in the torcetrapib/atorvastatin group compared with the atorvastatin monotherapy group. Subsequent to RADIANCE 1, the RADIANCE 2 study examined the effect of addition of torcetrapib to atorvastatin therapy on atherosclerosis progression, as measured by CIMT in patients with mixed dyslipidemia. ${ }^{31}$ Patients entering the study were 
required to have triglycerides $>151 \mathrm{mg} / \mathrm{dL}$ and LDL that merited statin therapy according to the NCEP ATP guidelines. Patients completed a 4-week washout period, in which all lipid-lowering therapy was discontinued, before entering an atorvastatin run-in phase. The atorvastatin dose was titrated to reach target LDL goals of $100 \mathrm{mg} / \mathrm{dL}, 130 \mathrm{mg} / \mathrm{dL}$, or $160 \mathrm{mg} / \mathrm{dL}$, according to individual CHD risk based on the NCEP guidelines. After completion of the run-in, patients $(\mathrm{N}=752)$ continued with atorvastatin therapy $(10,20,40$, or $80 \mathrm{mg}$ ) and were randomized to receive either torcetrapib $60 \mathrm{mg}$ or placebo. ${ }^{28}$ After 24 months of treatment, patients in the torcetrapib/atorvastatin group had a $63 \%$ increase in HDL and a $18 \%$ decrease in LDL compared with patients receiving atorvastatin monotherapy $(P<0.0001)$. Once again, the patients in the torcetrapib/atorvastatin group exhibited a $6.6 \mathrm{mmHg}$ elevation in systolic blood pressure compared with a $1.5 \mathrm{mmHg}$ elevation in the statin monotherapy group $(P<0.0001)$. Despite significant favorable improvements in LDL and HDL in the torcetrapib/atorvastatin group, change in maximum CIMT was not significantly different in the torcetrapib/atorvastatin group $(\mathrm{x}=0.025 \mathrm{~mm} /$ year $)$ compared with the atorvastatin monotherapy group $(\mathrm{x}=0.030 \mathrm{~mm} / \mathrm{year}$, $P=0.46) .{ }^{31}$

In summary, the addition of torcetrapib to background atorvastatin therapy did not have a significant effect on atherosclerosis progression as measured by vascular imaging techniques in the ILLUSTRATE, RADIANCE 1, and RADIANCE 2 trials, despite substantial increases in HDL. Also, it tended to increase the blood pressure in the treated group in all the three studies.

\section{Torcetrapib and clinical outcome trial (ILLUMINATE Study)}

The Investigation of Lipid Level Management to Understand its Impact in Atherosclerotic Events (ILLUMINATE) Trial sought to examine the effects of the addition of torcetrapib $60 \mathrm{mg}$ daily to baseline atorvastatin therapy in CHD patients who were at high risk according to NCEP criteria. ${ }^{32} \mathrm{~A}$ total of 15,067 participants between 45 and 75 years of age with high risk features including prior history of MI, stroke, acute coronary syndrome, unstable angina, peripheral vascular disease or type 2 diabetes first received atorvastatin titrated at 2-week intervals to reduce LDL level below $100 \mathrm{mg} / \mathrm{dL}$, and subsequently, they were randomized to either continuation of atorvastatin monotherapy or additional torcetrapib $60 \mathrm{mg} /$ day on top of the atorvastatin treatment. The primary end point was the time to occurrence of a first major CHD event, such as death from CHD due to MI, heart failure, or sudden cardiac death; non-fatal MI; stroke or unstable angina. At 1-year follow-up, the torcetrapib group showed a $72 \%$ rise in the level of HDL, along with $25 \%$ LDL and a $9 \%$ TG reduction compared with the atorvastatin only group $(P<0.001) .{ }^{32}$

Unfortunately though, the study was prematurely terminated due to an increased risk of death and major CHD events in the torcetrapib/atorvastatin group notwithstanding the positive effects on the lipid profile. As described above for the ILLUSTRATE and the RADIANCE studies, once again, the torcetrapib cohort showed a $5.4 \mathrm{mmHg}$ elevation in blood pressure above baseline $(P<0.001)$. Furthermore, higher mortality rate was experienced by the patient population in the torcetrapib arm. Overall, there were 93 deaths in the torcetrapib/atorvastatin group compared with only 59 deaths in the atorvastatin only group $(\mathrm{HR}=1.58 ; P=0.006)$. The HR for major CHD events was 1.25 in those treated with torcetrapib plus atorvastatin compared with those on atorvastatin monotherapy alone $(P=0.001)$.

\section{Ramifications of the results of the torcetrapib trials}

If all the four phase III torcetrapib studies are considered together including the results of the ILLUMINATE, ILLUSTRATE, RADIANCE 1, and RADIANCE 2 studies, it becomes clear that the adverse effects heavily outweighed any potential benefit emanating from raising the HDL levels. ${ }^{29-32}$ The lack of efficacy in terms of no demonstrable improvement in the parameters of atherosclerosis coupled with higher incidence of undesirable effects, such as higher rates of death, angina, revascularization procedures, and heart failure in the ILLUMINATE trial, and an increase in blood pressure in all 4 studies suggests a possible relationship to the mechanism of action of torcetrapib or CETP inhibitors as a class. Although clinical trials with torcetrapib were unsuccessful, it remains to be seen whether other CETP inhibitors would have beneficial effects on atherosclerosis without the above mentioned adverse effects; some such agents are currently being tested in randomized trials.

\section{New CETP inhibitors and trials in progress}

A trial by Roche Pharmaceuticals (dal-OUTCOMES) using an agent similar to torcetrapib in high risk patients with low baseline HDL is underway. This drug (dalcetrapib) reportedly does not raise blood pressure or aldosterone. The study's results should be available within a few years.

\section{Intravenous HDL agonists (apo A-I subtypes)}

Apo A-1 is the main lipoprotein associated with the HDL and it is secreted in the liver as well as in the intestine. Similar 
to HDL, apo A-1 levels correlate well with HDL levels. Also, apo A-1 corresponds inversely with the extent of atherosclerosis. Physiologically, the apo A-1 molecule acts by promoting the cellular cholesterol efflux process. It is also a major activator of the enzyme LCAT. As described above, apo A-1 is initially secreted as a lipid-void apo A-1 molecule (pre-HDL particle) and the lipid component is subsequently added to it in the presence of transport protein, such as ABCA-1 (lipidation). Studies in animal models have shown benefits of directly infusing synthetic apo A-1 models have opened a new therapeutic avenue for raising HDL. Apo A-1 overexpression in transgenic mice and rabbits has been demonstrated to increase the number of circulating HDL particles and confer protection from the development of diet- or gene-induced atherosclerosis.

Studies in mice and rabbits have used several approaches to increase HDL levels, including intravenous administration of synthetic HDL, apo A-1, and apo A-1 Milano and have demonstrated significant capacity to induce atheromatous plaque regression.

While carriers of the regular apo A-1 Milano show severe hypoalphalipoproteinemia but no increase in the risk for premature $\mathrm{CHD}$, an apo A-1 Milano variant produced by substituting cysteine for arginine at position 173 makes the molecule quite protective. Such substitution allows more effective lipidation and genesis of large HDL particles with longer serum half-life. The HDLs derived from patients with the apoA-1 ${ }_{\text {Milano }}$ phenotype have enhanced affinity for ABC A-1 and augmented ability for reverse cholesterol transport process. Apo A-1 Milano also exhibits greater affinity for ABCA-1 and more pronounced capacity to antagonize lipid oxidation and thrombosis as compared with native apo A-1. It is, therefore, reasonable to assess the therapeutic utility of direct intravenous infusion of the apo A-1 variants in the setting of established CHD.

\section{ETC 216 study (intravenous apo A-I Milano/phospholipid complex infusion)}

The effect of once-weekly intravenous infusion was studied by Nissen et al in 123 patients with established CHD using recombinant apo A-1 Milano/ 1-palmitoyl-2-oleoyl phosphatidylcholine complex (ETC 216). Efficacy on the atheroma burden was measured by IVUS both at baseline and at 5 weeks. A significant regression of coronary atherosclerosis was demonstrated as indicated by change in percent atheroma volume $-4.2 \%$ versus $-0.8 \%$ [ $P<0.001]$ in patients treated with ETC 216 and placebo respectively. The mean change in the total atheroma volume was $-14.1 \mathrm{~mm}^{3}$ versus $-2.9 \mathrm{~mm}^{3}[P<0.001]$ in patients assigned to ETC 216 and placebo respectively. ${ }^{29}$

\section{ERASE study}

In the Atherosclerosis Safety and Efficacy (ERASE study), 183 patients were randomized to receive placebo or one of the two dosages, $40 \mathrm{mg} / \mathrm{kg}$ or $80 \mathrm{mg} / \mathrm{kg}$, of CSL-111 intravenously for a 4-week period. The molecule CSL-111was produced as a reconstituted HDL particle consisting of apo A-1 from human plasma combined with soybean phosphatidylcholine, which chemically and biologically resembles native HDL. Plaque characterization by IVUS and quantitative coronary angiography was performed at baseline and at 4 weeks that showed significant improvement in the plaque characteristics in the treated groups as compared with placebo. However, no significant differences were found in the atheroma volume. Also, the higher dosage of CSL-3 treatment $(80 \mathrm{mg} / \mathrm{kg})$ had to be discontinued early because of the development of liver function abnormalities.

Thus, although infusible HDL agonists seem to provide benefit in the short term as shown by plaque regression through IVUS assessment, much more work must be done to better characterize the potential efficacy of these therapies in both the near and long term to see if clinical outcome is influenced in the patients at high risk. In addition, safety concerns, such as anaphylactic reactions, liver dysfunction, and cost implications will quite likely impact further testing and usage. One intriguing possibility may be that such an approach to HDL modulation by treating it in acute situations may offer means by which to stabilize vulnerable atheromatous plaques and decrease their propensity to rupture and cause acute coronary events.

\section{Niacin and HDL}

\section{Studies with niacin}

Niacin, also called nicotinic acid, is one of the water-soluble $\mathrm{B}$ vitamins. It favorably modifies most of the lipid subfractions, including raising the HDL levels. Niacin acts by binding to the surface of the adipocytes through a receptor labeled HM74 (or gp 109A). This binding inhibits hormonesensitive lipase that results in decreased mobilization of fatty acids into the portal circulation. Additionally, niacin decreases the formation of triglycerides by inhibiting the enzyme, acyl-coenzyme A: diglycerol acyltransferase 2 (DGAT2) that esterifies mono- and diglycerides to form triglycerides. Niacin also increases apo-B100 catabolism causing reduced VLDL and triglyceride secretion by the liver. Niacin raises serum HDL levels by decreasing HDL 
catabolism in the liver by down-regulating the expression of the F1 moiety of F1F0 ATP synthetase, a holoparticle HDL receptor. Another mechanism by which niacin raises HDL-C level may include the reduced enrichment of HDL with triglyceride and its subsequent catabolism by hepatic lipase enzyme.

Niacin is currently the most effective pharmacotherapeutic agent approved and available for raising HDL-C. ${ }^{33}$ It has an established safety profile and has been used for over 50 years. According to ADA and NCEP, niacin can safely be used even in patients with diabetes. Lately, it has been tested and found efficacious in combination therapy with statins to simultaneously reduce LDL and raise HDL. ${ }^{34}$ The coronary drug project (CDP), a placebo-controlled trial conducted 3 decades ago, randomized 6341 men with prior history of MI, to evaluate the effectiveness of 5 different lipid-altering agents that included clofibrate, dextrothyroxine, low- and high-dose estrogen and niacin. Due to undesirable side effects estrogen and dextrothyroxine groups were prematurely stopped, while the niacin and clofibrate groups completed the 6.2-year study. Even though the total mortality was not significantly different in the niacin group compared with the placebo group (24.4\% and $25.4 \%$ respectively), significant reduction was seen in the niacin cohort in the combined outcome of CHD death and MI $(15 \%, P<0.05)$, non-fatal MI $(26 \%, P<0.05)$, cerebro-vascular events $(24 \%, P$ $<0.05)$, and need for cardiac surgery $(47 \%, P<0.05) .{ }^{35}$ Nine years following the termination of the CDP trial, a 15-year follow-up was performed and a remarkable finding was made. Reviewers now found that, at 15 years, mortality reduction had become significant in the niacin group. Compared with the placebo group, the niacin group experienced an $11 \%$ reduction in total mortality and $12 \%$ reduction in CHD mortality $\left(P=0.0004\right.$ and $P<0.05$, respectively. ${ }^{36}$ These findings suggest that treatment with niacin increased survival and the benefit was maintained even after stopping the therapy.

\section{Improving niacin compliance}

Compliance is a significant issue because of the significant frequency with which patients experience flushing as a side effect and opt to discontinue niacin. It has been often noted that when physicians sit down with patients and discuss the benefits and nuances of niacin, patient compliance goes up. In fact, the significance of physician support in increasing compliance to niacin was evaluated in the Impact of Medical Subspecialty on Patient Compliance to Treatment (IMPACT) study. ${ }^{37}$ The researchers concluded that long-term adherence to niacin therapy is greatly dependent on patient education and awareness. The physicians and supporting health team members should discuss with the patients directly about the side effect of flushing and the high possibility of development of tolerance over time if the treatment was continued without interruption, which can prevent tolerance from developing.

Several formulations of extended release niacin that seem to have much less problem of flushing as well as higher rates of tolerance with continued use have been developed and studied over the past decade. In a study, niacin ER was in doses of up to $3 \mathrm{~g}$ daily was evaluated in 517 patients who were followed for about 10 months. Over the course of the study, the number of patients who reported the episodes of flushing progressively declined by $90 \%$. Most patients experienced 1 mild flush per month on an average. ${ }^{38}$ Strategies to reduce flushing include several simple measures. Such measures may include (a) use of niacin ER once daily at bedtime, (b) taking niacin with aspirin or another nonsteroidal anti-inflammatory agent taken approximately 30 minutes prior, (c) gradual increase in the dose, (d) taking niacin with a low-fat, high-fiber snack, and (e) avoiding triggers such as heat or ingestion of alcohol or spicy foods. ${ }^{39} \mathrm{~A}$ new formulation of niacin ER that is now available has shown a significant $42 \%$ to $43 \%$ decrease in median severity and duration of flushing $(P<0.0001)$ as compared with the old formulation. ${ }^{40}$ Furthermore, Cefali et al used the niacin ER (2000 mg) and tested the effectiveness of $650 \mathrm{mg}$ aspirin on flushing when the aspirin was given 30 minutes prior to niacin therapy, concomitant with niacin therapy, or not at all. Both, pre- and concomitant treatments with aspirin, exhibited $42 \%$ reduction in median severity of flushing as compared with the new formulation of niacin ER without aspirin $(P<0.001){ }^{41}$

\section{Cordaptive (extended-release niacin and PGDI receptor antagonist)}

Niacin raises the HDL by $15 \%$ to $30 \%$. Despite its ability to significantly increase the HDL levels, the use of niacin in the treatment of low HDL has been limited due to its side-effect of flushing. The flushing is caused by cutaneous vasodilation leading to warmth and itching. Such vasodilatation is mediated by activation of the formation of prostaglandin D2 (15-deoxyD12,14-prostaglandin J2), which binds to the prostaglandin D2 receptor 1 (PGD1 receptor) in the skin. Therapeutic agents that can block the PGD1 receptor may have the potential to reduce the prostaglandin D2 production and subsequent cutaneous flushing. Laropiprant is one such agent under investigation. 
A novel therapeutic agent, Cordaptive, previously known as MK-0524A, is a combination of extended-release niacin and a PGD1 receptor blocker (laropiprant), which functions to prevent activation of vasodilatation by prostaglandin D2. ${ }^{42}$ It has been shown in limited clinical studies to reduce symptoms of flushing. Cordaptive is already approved for use in the European Union but not in the US. Cordaptive is now being studied in ACHIEVE and HPS2-THRIVE (the Treatment of HDL to Reduce the Incidence of Vascular Events) studies. These trial data may lead to its approval in the US if the benefit/risk ratio comes out to be favorable.

A word of caution about Cordaptive or laropiprant is, however, in order. One of the metabolites of prostaglandin D2 is a potent endogenous PPAR- $\gamma$ agonist that promotes expression of ABCA-1 and increases transfer of cholesterol from macrophages to HDL. Cordaptive, by reducing prostaglandin D2 production, thus, may potentially cause an undesirable side effect of decreasing the ability of prostaglandin D2 to stimulate expression of ABCA-1 on the surface of macrophages.

\section{Aggressive statin monotherapy for raising HDL}

There are substantial data available in the literature from multiple randomized clinical trials proving beyond doubt that statins significantly reduce LDL and improve clinical outcomes. HDL is usually only modestly raised by using the statins in higher doses as part of aggressive statin monotherapy. Several trials incorporating angiography, IVUS or measurement of CIMT have demonstrated that treatment with aggressive statin monotherapy that aggressively lowers LDL and modestly raises HDL (by $10 \%$ to $15 \%$ ) significantly reduces the progression of atherosclerosis. The Arterial Biology for the Investigation of the Treatment Effects of Reducing Cholesterol (ARBITER) study explored whether bringing LDL to much below $100 \mathrm{mg} / \mathrm{dL}$ would decrease atherosclerosis progression as measured by CIMT in 161 patients treated for 12 months. The LDL decreased to a mean of $76 \mathrm{mg} / \mathrm{dL}$ with atorvastatin $80 \mathrm{mg}$ in the aggressive therapy group and showed significant concomitant CIMT regression $(-0.034 \mathrm{~mm})$ as compared with pravastatin group $(+0.025 \mathrm{~mm}, P=0.03){ }^{43}$

The Measuring Effects on Intima-Media Thickness: an Evaluation of Rosuvastatin (METEOR) study assessed the effect of very high-intensity statin therapy on CIMT over 2 years. The patients in the rosuvastatin group experienced a $49 \%$ reduction in LDL (mean, $78 \mathrm{mg} / \mathrm{dL}$ ) along with significantly slowed progression of atherosclerosis as measured by CIMT $(-0.0014 \mathrm{~mm} /$ year) compared with placebo $(+0.0131 \mathrm{~mm} /$ year, $P<0.001) .{ }^{44}$ These studies showed slowed progression but did not show significant atheroma regression.

Regression of atherosclerosis was demonstrated in A Study to Evaluate the Effect of Rosuvastatin on Intravascular Ultrasound-Derived Coronary Atheroma Burden (ASTEROID) examined the effects of high-dose rosuvastatin on progression of coronary atherosclerosis. The mean LDL was reduced by $53 \%$ to $60.8 \mathrm{mg} / \mathrm{dL}(P<0.00)$ and the mean HDL was increased by $15 \%$ to $49.0 \mathrm{mg} / \mathrm{dL}(P<0.001) .{ }^{45}$ The primary efficacy parameter of mean percent atheroma volume (PAV) was reduced from $39.6 \%$ to $38.6 \%$, $(-0.98 \%$, $P<0.001)$. For this measure, the mean percent atheroma volume, $63.6 \%$ of patients experienced regression. If total atheroma volume was used as the parameter and applied to only the subsegment with greatest disease severity, $78.1 \%$ of patients exhibited disease regression. When quantitative coronary angiography (QCA) was used for the same patients, the mean percent diameter stenosis was reduced from $37.3 \%$ to $36.0 \%$ after 24 months of rosuvastatin $40 \mathrm{mg}$ therapy $(P<0.001){ }^{46}$

\section{Combination therapies for raising HDL HDL-Atherosclerosis Treatment Study (HATS)}

In HATS, simvastatin and niacin combination therapy was compared with placebo in CHD patients with low $\mathrm{HDL}$ ( $\leq 35 \mathrm{mg} / \mathrm{dL}$ in men or $\leq 40 \mathrm{mg} / \mathrm{dL}$ in women) and LDL-C levels $\leq 145 \mathrm{mg} / \mathrm{dL}$ and followed them for 3 years in a double-blind fashion. ${ }^{47}$ Patients in combination therapy group experienced a $42 \%$ reduction in LDL, a $36 \%$ reduction in TG, and a $26 \%$ elevation of HDL $(P<0.001)$ over their baseline values. While patients who received placebo showed progression of the most severe coronary stenosis $(+3.9 \%)$, the patients who received the combination therapy exhibited regression $(-0.4 \%, P<0.001)$. Clinical outcome was significantly improved as well in the form of a striking 90\% reduction in the CHD events in patients receiving simvastatin and niacin combination therapy compared with patients who received placebo $(P=0.03)$.

\section{Familial Atherosclerosis Treatment Study (FATS)}

FATS examined the efficacy of combination therapy using either lovastatin and colestipol, niacin and colestipol, or placebo in addition to dietary counseling as measured by QCA in men with CHD and a family history of vascular disease. In patients treated with combination therapies, such as lovastatin and colestipol and niacin and colestipol, the LDL was reduced by $46 \%$ and $32 \%$, respectively $(P<0.001$ versus baseline) and HDL was increased by $15 \%$ and $43 \%$, 
respectively ( $P<0.01$ and $P<0.001$ versus baseline). In contrast, the placebo group merely showed $7 \%$ decline in the LDL and 5\% rise in the HDL. Among those patients receiving placebo, $46 \%$ showed lesion progression and $11 \%$ showed lesion regression in at least 1 of 9 proximal coronary segments evaluated. ${ }^{48}$ In comparison, patients receiving lovastatin and colestipol or niacin and colestipol had less frequent lesion progression (21\% and 25\%, respectively) and significantly more frequent lesion regression (lovastatin and colestipol, 32\%; niacin and colestipol, 39\%; $P<0.005$ versus placebo). Clinical events, such as death, MI, or revascularization were similarly influenced. While $19.2 \%$ patients in the placebo group experienced a CHD event, only $6.5 \%$ of the patients did so in the lovastatin and colestipol group and $4.2 \%$ of patients in the niacin and colestipol group.

In a Follow-up study of FATS trial, 75 patients on triple combination therapy with niacin, colestipol, and lovastatin were followed further. At 10 years, approximately $19.8 \%$ of the patients in the control group died as compared with only $1.3 \%$ of the patients in the triple therapy group (relative risk reduction $=93 \%, P<0.001$ ). In addition, $18.8 \%$ of the control group experienced a composite endpoint of death or MI compared with $5.3 \%$ of the patients in the triple therapy group (relative risk reduction $=67 \%, P<0.02$ ). ${ }^{49}$

Arterial Biology for the Investigation of the Treatment Effects of Reducing Cholesterol trials:ARBITER-2 and ARBITER-3 (niacin ER and statin).

Combination therapy with niacin formulations and statin was evaluated in the ARBITER-2 and ARBITER-3 trials. In the ARBITER-2, a year-long treatment with a combination regimen involving extended-release niacin and atorvastatin was given to patients with known CHD and low HDL (defined as $<45 \mathrm{mg} / \mathrm{dL}$ ) in a double-blind, randomized manner with placebo control. ${ }^{50}$ The primary end point of the study was the change in CIMT from baseline after 12 months of treatment. While there were no significant changes seen in the LDL levels; the HDL levels significantly increased by $21 \%$ and TG levels significantly declined by $13 \%(P=0.003$ and $P=0.03$ respectively $)$. The CIMT increased significantly by $+0.044 \mathrm{~mm}$ in the statin monotherapy (control) group ( $P<0.001$ versus baseline). In contrast the increase was much less, ie, $+0.014 \mathrm{~mm}$, and it remained non-significant in the combination therapy group ( $P=0.23$ versus baseline).

The ARBITER-3 trial was an extension study of ARBITER 2 that permitted patients in the placebo (statin monotherapy) group to cross over to niacin ER and statin combination regimen. These crossed over patients exhibited significant $12 \%$ drop in LDL $(P=0.012), 33 \%$ drop in TG $(P=0.001)$ and $24 \%$ rise in HDL $(P<0.001)$ after 2 years of niacin ER therapy. Furthermore, at 1- and 2-year points following the start of combination therapy, regression in atherosclerosis was noted by CIMT $(-0.027 \mathrm{~mm}$ and $-0.041 \mathrm{~mm}$, respectively; $P<0.001$ versus statin monotherapy). ${ }^{51}$ High-risk patients with diabetes or the metabolic syndrome who were randomized to combination therapy, experienced somewhat greater regression of CIMT $(-0.046 \mathrm{~mm}, P<0.001)$. More interestingly, a statistically significant inverse correlation between the increase in HDL and the regression of CIMT $(P=0.002)$ was noted suggesting that raising HDL with niacin ER over and above the statin treatment may account for the beneficial effect of atherosclerosis regression encountered.

\section{Combination therapy and CHD risk reduction: summary}

A recent meta-analysis compared statin monotherapy trials and combination lipid-modifying therapy trials using clinical event relative risk reduction and the number needed to treat (NNT to prevent 1 CHD event. ${ }^{52}$ These studies had diverse patient populations, numbers of subjects, and treatment durations, which merits consideration when interpreting the results.

The six clinical end point LDL-lowering trials included the Scandinavian Simvastatin Survival Study (4S), Long-Term Intervention with Pravastatin in Ischemic Disease (LIPID), Cholesterol And Recurrent Events Trial (CARE), West of Scotland Coronary Prevention Study Group (WOSCOPS), Air Force/Texas Coronary Atherosclerosis Prevention Study (AF/TexCAPS), and Collaborative Atorvastatin Diabetes Study (CARDS). These achieved an average clinical event relative risk reduction of $27.2 \%$ and an average NNT per year of 8.5. In addition, the three recent, intensive LDL-lowering clinical end point trials (IDEAL, TNT, and PROVE IT) achieved an average clinical event relative risk reduction of $16.0 \%$ and an average NNT per year of 19.1 . Furthermore, five angiographic LDL- lowering trials that include Monitored Atherosclerosis Regression Study (MARS), Multicentre Anti-Atheroma Study (MAAS), Regression Growth Evaluation Statin Study (REGRESS), Pravastatin Limitation of Atherosclerosis in the Coronary Arteries (PLAC-I), and Canadian Coronary Atherosclerosis Intervention Trial (CCAIT), achieved an average clinical event relative risk reduction of $29.6 \%$ and an average NNT per year of 28.7 . 
In contrast, clinical trials that tested combination therapies for simultaneously lowering LDL and raising HDL, such as FATS, FATS- long follow-up, Cholesterol Lowering Atherosclerosis Study (CLAS), CLAS- 2-year follow-up, HATS, and Stanford Coronary Risk Intervention Project (SCRIP), exhibited much higher efficiency. They achieved an average clinical event relative risk reduction of $71.6 \%$ and an average NNT per year of only 3.4. These data suggest enhanced efficiency for reducing clinical events using LDL-lowering plus HDL-raising therapy versus LDL-lowering therapy alone.

In 2007, a National Lipid Association (NLA) task force examined the safety of niacin therapy and concluded that 2 decades of clinical evidence does not show an adverse augmentation of myopathy by niacin whether used alone or in combination with statins. Therefore, the addition of niacin to statin monotherapy appears to be a safe method for improving multiple atherogenic lipid abnormalities including raising the HDL in high risk CHD patients. Niacin should, however, be prescribed to patients as such or in the extended-release form. Some of the niacin formulations found in health food stores has been shown to cause significant hepato-toxicity which is not seen with the prescription forms of niacin ER. ${ }^{53}$ In fact, the use of such unregulated niacin preparations has been discouraged by the AHA.

\section{Future HDL-raising therapies}

PPAR- $\gamma$ agonists

Some of the currently available dyslipidemic agents that include the fibric acid derivatives, such as gemfibrozil, fenofibrate, and bezafibrate, are PPAR- $\gamma$ agonists. These agents have not been that potent, however. Some of the newer more potent agents are currently undergoing trials that hold significant promise for use as future HDL-raising agents. The PPAR- $\gamma$ is one of the several PPAR isoforms that include PPAR $-\gamma$, PPAR- $\beta / \sigma$, and PPAR- $\gamma$. The PPARs are nuclear transcription factors. PPAR isoforms have varying properties that depend on their target genes and tissue distribution. For example, while the PPAR- $\gamma$ regulates genes involved in fatty acid uptake and storage, inflammation, and lipoprotein metabolism, the PPAR- $\gamma$ regulates genes involved in glucose and fatty acid metabolism, inflammation, and macrophage lipid homeostasis. ${ }^{54}$

The activation of PPAR $-\gamma$ causes rise in the serum HDL level by several mechanisms that include stimulation of hepatic apo A-1 expression, promotion of TG catabolism by activating lipoprotein lipase, decreasing the enrichment of HDL with TG and eventually reducing the lipolysis and catabolism of HDL by hepatic lipase. ${ }^{55}$ Furthermore, the hepatic apo A-1 and apo A-2 expression is increased on activation of PPAR $-\gamma$. The activation of the PPAR- $\gamma$ also augments the cholesterol efflux, accelerates RCT and raises the HDL level. The PPAR- $\gamma$ agonists act to affect atherosclerosis by several additional mechanisms including improving vascular wall reactivity, reducing expression of endothelial adhesion molecules, and decreasing levels of inflammatory cytokines such as tumor necrosis factor- $\alpha$ (TNF- $\alpha)$, interleukin (IL)-I, and IL-6.

Clinically the fibric acid derivatives act to decrease plasma TGs and VLDL and increase HDL. They are, therefore, recognized as first-line agents in the treatment of primary hypertriglyceridemia, combined hyperlipidemia, and secondary lipid abnormalities including mixed dyslipidemias. While fibrates are PPAR- $\gamma$ agonists, they demonstrated only weak agonist activity. They have to be used in high doses, such as 145 to $1200 \mathrm{mg}$ daily to achieve desired lipid-lowering effects.

Currently, somewhat more potent and subtype-selective PPAR- $\gamma$ agonists are under development. ${ }^{56}$ They include GFT505, a selective PPAR- $\gamma$ modulator that is 100-times more potent than fenofibrate. There are several other new selective PPAR $-\gamma$ agonists in the pipeline that may prove beneficial in improved regulation of lipid metabolism as well as augmentation of HDL levels.

\section{Liver $X$-receptor- $\alpha$ agonists}

LXRs are also nuclear transcription factors that are involved in the regulation of lipid metabolism and regulation of inflammation. They have the potential of raising HDL levels because they up-regulate the ABCA-1 and ABCG-1 receptors in the macrophages that promote RCT. ${ }^{57}$ Thiazolidinediones are molecules that activate LXR- $\alpha$ and increase expression of ABCA-1 and ABCG-1 but they are not therapeutically usable as such. Synthetic LXR agonists that are currently under development cause regression of atherosclerosis and enhance macrophage cholesterol efflux and RCT process in the animal models. They were shown to inhibit the progression of atherosclerosis in LDL receptor knockout and apo-E knockout mice., LXR agonists also inhibit lipopolysaccharide-induced expression of proinflammatory genes and decrease inflammation in mouse models.

There is a potential negative side to this approach. The therapeutic benefit of LXR agonists could be diminished because of LXR-mediated increases in plasma and hepatic triglycerides resulting from induction of lipogenic genes, including sterol response element binding protein-1c 
(SREBP-1c) and the enzyme, fatty acid synthase. This can lead to increased risk of developing hepatic steatosis.

Several of the new LXR agonists are currently undergoing evaluation. One such synthetic agent, GW3965, was found to increase the rate of RCT in vivo without inducing hepatic steatosis. Continued interest in LXR agonism as a potential therapeutic approach may one day lead to more selective LXR modulators with lesser tendency for hepatic steatosis. $^{58}$

\section{D-4F and other apo-A like agents}

The apoprotein subunit of the apoipoprotein contains $\alpha$-helices that are n-amphipathic and help stabilize the structure and size of lipoproteins. These helical regions in the apolipoprotein of the HDL, apo A-1, similarly allows HDL to dock with the cell surface receptors ABCA-1 and SR-BI. The apoprotein agonists may have the potential to be used as therapeutic agents for augmenting HDL. However, one must note that the apoprotein agonists are amphipathic $\alpha$-helices that do not necessarily raise the serum levels of HDL in quantity. Instead, such agents augment the functional quality or overall effectiveness of HDL-C.

One of the apo A-I agonists, D-4F, is a molecule that is resistant to hydrolysis by gastric peptidases because it is composed of D-amino acids with 4 phenyl-alanine residues in its amino acid sequence, and can be taken orally. D-4F has been shown to enhance the antioxidant and anti-inflammatory properties of HDL, and accelerate the RCT process. ${ }^{59}$ The antiatherogenic effect of $\mathrm{D}-4 \mathrm{~F}$ has been demonstrated in hyperlipidemic mice and in apolipoprotein E-null mice. When given orally to mice and monkeys, D-4F leads to greater production of pre- $\beta$ HDL, and improved HDLmediated cholesterol efflux from macrophages to some extent. More importantly, D-4F helps convert proinflammatory HDL to anti-inflammatory HDL. ${ }^{60}$

In a study, D-4F, when orally administered to the LDL receptor-null mice that were fed Western diet and apoE-null mice that were fed chow diet, demonstrated decrease in the atherosclerotic lesions by $79 \%$ and $75 \%$, respectively, without significantly altering the plasma lipid levels. ${ }^{61}$ Thus, the studies using D-4F are intriguing; however, we have a long way to go before its optimal use, systemic effects and safety profile are fully worked out. If results come out favorable, the D-4F may become quite a cost-effective agent for use as an apo A-1 agonist for patients with low HDL levels or dysfunctional RCT. A number of additional apo A-1 agonist peptides are also in development at the present time and hold promise for HDL therapy.

\section{Farnesoid $X$ receptor (FXR) antagonists}

The farnesoid $\mathrm{X}$ receptor (FXR) molecule belongs to the nuclear receptor family. FXR participates in the regulation of bile acid synthesis, conjugation, and transport. ${ }^{62}$ It also enhances the expression of one of the enzymes, phospholipid transfer protein, involved in HDL formation, and lowers TG levels by inducing PPAR- $\alpha$ that promotes clearance. One of the most important functions of FXR activation is to inhibit cholesterol 7-alpha-hydroxylase, the rate-limiting enzyme in bile acid biosynthesis. FXR is expressed in a wide variety of tissues, including the heart, kidney, thymus, and spleen, and vasculature. The role of FXR in HDL metabolism is unclear, however, the apoA-I and hepatic lipase expression are both inhibited by activated FXR. Thus, antagonizing FXR function to favorably influence HDL metabolism and RCT is intriguing. Studies on the use of guggolipid as an antagonist of FXR have not demonstrated consistent results in raising HDL. ${ }^{63}$ Therefore, much more research is needed to establish what role FXR plays in the HDL metabolism and what if any role FXR modulators have in CHD prevention in future.

\section{Conclusions}

Despite significant reductions in CHD events with statin therapy, significant proportion of residual events still occur, and they are unacceptable. HDL is a significant, independent risk factor that has been suggested as the next therapeutic target for reducing residual CHD risk. There is an inverse relationship between HDL and CHD risk that may be attributed to the role of HDL in promoting reverse cholesterol transport process and imparting additional antiatherogenic actions. In recognition of the significant risk associated with low HDL levels, national guidelines now support raising HDL to reduce CHD risk once LDL goals are met. Currently, the recommended therapies for raising HDL include lifestyle modification, and use of niacin, fibrates or combination therapeutic agents. Although statin therapy has an established efficacy in increasing HDL levels, only minor overall increases in HDL have been demonstrated. Multiple new agents that affect HDL metabolism are undergoing trials, and the outcomes of these trials may lead to better therapies in future. Thus, it is likely that the modulation of HDL levels or its properties will significantly impact future approaches to the management of cardiovascular disease in both the primary and secondary prevention settings.

These promising therapies include therapeutic adjustment of enzymes that remodel HDL and facilitate transfer reactions among lipoproteins, such as CETP, phospholipid transfer protein, endothelial lipase, lipoprotein lipase, and hepatic 
lipase. In addition, the Infusible apoproteins and HDL, apo A-1 agonists, nuclear transcription factor agonists, and macrophage-surface $\mathrm{ABC}$ transporters will also find a role in HDL modulation. Certainly, well-designed clinical studies and large clinical trials will be needed to demonstrate both safety and efficacy given the difficulties and lessons learned from the torcetrapib trials rather than mere demonstration of atherosclerosis modification through IVUS, or CIMT.

\section{Disclosure}

The authors declare no conflicts of interest.

\section{References}

1. Rosamond W, Flegal K, Furie K, et al. Heart disease and stroke statistics - 2008 update: a report from the American Heart Association Statistics Committee and Stroke Statistics Subcommittee. Circulation. 2008;117:e25-e146.

2. Llloyd-Jones DM, Hong Y, Labarthe D, et al. Defining and setting national goals for cardiovascular health promotion and disease reduction: The American Heart Association's strategic impact goal through 2020 and beyond. Circulation. 2010;121:586-613.

3. Cannon $\mathrm{CP}$, Braunwald $\mathrm{E}, \mathrm{McCabe} \mathrm{CH}$, et al. Intensive versus moderate lipid lowering with statins after acute coronary syndromes. $N$ Engl J Med. 2004;350:1495-1504.

4. Pedersen TR, Faergeman O, Kastelein JJ, et al. High-dose atorvastatin vs usual-dose simvastatin for secondary prevention after myocardial infarction: the IDEAL study: a randomized controlled trial. JAMA. 2005;294:2437-2445.

5. LaRosa JC, Grundy SM, Waters DD, et al. Intensive lipid lowering with atorvastatin in patients with stable coronary disease. $N$ Engl $J$ Med. 2005;352:1425-1435.

6. Third Report of the National Cholesterol Education Program (NCEP) Expert Panel on Detection, Evaluation, and Treatment of High Blood Cholesterol in Adults (Adult Treatment Panel III) final report. Circulation. 2002;106:3143-3421.

7. Gordon DJ, Probstfield JL, Garrison RJ, et al. High-density lipoprotein cholesterol and cardiovascular disease. Four prospective American studies. Circulation. 1989;79:8-15.

8. Ford ES, Giles WH, Dietz WH. Prevalence of the metabolic syndrome among US adults: findings from the third National Health and Nutrition Examination Survey. JAMA. 2002;287:356-359.

9. Castelli WP. Cholesterol and lipids in the risk of coronary artery disease - the Framingham Heart Study. Can J Cardiol. 1988;4 Suppl A $5 \mathrm{~A}-10 \mathrm{~A}$

10. Baigent C, Keech A, Kearney PM, et al. Efficacy and safety of cholesterol-lowering treatment: prospective meta-analysis of data from 90,056 participants in 14 randomised trials of statins. Lancet. 2005;366: 1267-1278.

11. van der Steeg WA, Holme I, Boekholdt SM, et al. High-density lipoprotein cholesterol, high-density lipoprotein particle size, and apolipoprotein A-I: significance for cardiovascular risk: the IDEAL and EPIC-Norfolk studies. J Am Coll Cardiol. 2008;51:634-642.

12. Barter P, Gotto AM, LaRosa JC, et al. HDL cholesterol, very low levels of LDL cholesterol, and cardiovascular events. $N$ Engl $J$ Med. 2007;357:1301-1310.

13. Lewis GF, Rader DJ. New insights into the regulation of HDL metabolism and reverse cholesterol transport. Circ Res. 2005;96:1221-1232.

14. Cuchel M, Rader DJ. Macrophage reverse cholesterol transport: key to the regression of atherosclerosis? Circulation. 2006;113: 2548-2555.

15. Hovingh GK, Hutten BA, Holleboom AG, et al. Compromised LCAT function is associated with increased atherosclerosis. Circulation. 2005;112:879-884.
16. Chapman MJ, Assmann G, Fruchart JC, Shepherd J, Sirtori C. Raising high-density lipoprotein cholesterol with reduction of cardiovascular risk: the role of nicotinic acid - a position paper developed by the European Consensus Panel on HDL-C. Curr Med Res Opin. 2004;20: 1253-1268.

17. Genest J. The Yin and Yang of high-density lipoprotein cholesterol. J Am Coll Cardiol. 2008;51:643-644.

18. Singh V, Deedwania P. Dyslipidemia in special populations: Asian Indians, African Americans and Hispanics. Curr Atheroscler Rep. 2006;8:32-40.

19. Yusuf S, Hawken S, Ounpuu S, et al. Effect of potentially modifiable risk factors associated with myocardial infarction in 52 countries (the INTERHEART study): case-control study. Lancet. 2004;364: 937-52.

20. Dodani S, Dong Y, Zhu H, George V. Can novel Apo A-I polymorphisms be responsible for low HDL in South Asian immigrants? Indian J Hum Genet. 2008;14:9-15.

21. Mosca L, Appel LJ, Benjamin EJ, et al. Evidence-based guidelines for cardiovascular disease prevention in women. Circulation. 2004;109: 672-693.

22. Mosca L, Banka CL, Benjamin EJ, et al. Evidence-based guidelines for cardiovascular disease prevention in women: 2007 update. J Am Coll Cardiol. 2007;49:1230-1250.

23. Grundy SM, Cleeman JI, Merz CN, et al. Implications of recent clinical trials for the National Cholesterol Education Program Adult Treatment Panel III guidelines. Circulation. 2004;110:227-239.

24. American Diabetes Association. Dyslipidemia management in adults with diabetes. Diabetes Care. 2004;27 Suppl 1:S68-S71.

25. American Diabetes Association. Standards of medical care in diabetes - 2008. Diabetes Care. 2008;31:S12-S54.

26. Brunzell JD, Davidson M, Furberg CD, et al. Lipoprotein management in patients with cardiometabolic risk: consensus statement from the American Diabetes Association and the American College of Cardiology Foundation. Diabetes Care. 2008;31:811-822.

27. Rader DJ. Illuminating HDL - is it still a viable therapeutic target? N Engl J Med. 2007;357:2180-2183.

28. Tall AR, Yvan-Charvet $L$, Wang $N$. The failure of torcetrapib: was it the molecule or the mechanism? Arterioscler Thromb Vasc Biol. 2007;27: $257-260$.

29. Nissen SE, Tardif JC, Nicholls SJ, et al. Effect of torcetrapib on the progression of coronary atherosclerosis. $N$ Engl J Med. 2007;356: 1304-1316.

30. Kastelein JJ, van Leuven SI, Burgess L, et al. Effect of torcetrapib on carotid atherosclerosis in familial hypercholesterolemia. $N$ Engl J Med. 2007;356:1620-1630.

31. Bots ML, Visseren FL, Evans GW, et al. Torcetrapib and carotid intima-media thickness in mixed dyslipidaemia (RADIANCE 2 study): a randomised, double-blind trial. Lancet. 2007;370:153-160.

32. Barter PJ, Caulfield M, Eriksson M, et al. Effects of torcetrapib in patients at high risk for coronary events. $N$ Engl J Med. 2007;357: 2109-2122.

33. Brown WV. Expert commentary: the safety of fibrates in lipid-lowering therapy. Am J Cardiol. 2007;99:19C-21C

34. Guyton JR, Bays HE. Safety considerations with niacin therapy. Am J Cardiol. 2007;99:22C-31C

35. The Coronary Drug Project Research Group. Clofibrate and niacin in coronary heart disease. JAMA. 1975;231:360-381.

36. Canner PL, Berge KG, Wenger NK, et al. Fifteen year mortality in Coronary Drug Project patients: long-term benefit with niacin. $J \mathrm{Am}$ Coll Cardiol. 1986;8:1245-1255.

37. Rubenfire M. Safety and compliance with once-daily niacin extendedrelease/ lovastatin as initial therapy in the Impact of Medical Subspecialty on Patient Compliance to Treatment (IMPACT) study. Am J Cardiol. 2004;94:306-311.

38. Capuzzi DM, Guyton JR, Morgan JM, et al. Efficacy and safety of an extended-release niacin (Niaspan): a long-term study. Am J Cardiol.1998;82:74U-81U; discussion 85U-86U.

39. McKenney J. New perspectives on the use of niacin in the treatment of lipid disorders. Arch Intern Med. 2004;164:697-705. 
40. Cefali EA, Simmons PD, Stanek EJ, Shamp TR. Improved control of niacin-induced flushing using an optimized once-daily, extended-release niacin formulation. Int J Clin Pharmacol Ther. 2006;44:633-640.

41. Cefali EA, Simmons PD, Stanek EJ, McGovern ME, Kissling CJ. Aspirin reduces cutaneous flushing after administration of an optimized extended-release niacin formulation. Int J Clin Pharmacol Ther. 2007;45:78-88.

42. Deedwania P, Singh V, Davidson MH. Low high-density lipoprotein cholesterol and increased cardiovascular disease risk: An analysis of statin clinical trials. Am J Cardiol. 2009;104 Suppl:3E-9E.

43. Taylor AJ, Kent SM, Flaherty PJ, Coyle LC, Markwood TT, Vernalis MN. ARBITER: Arterial Biology for the Investigation of the Treatment Effects of Reducing Cholesterol: a randomized trial comparing the effects of atorvastatin and pravastatin on carotid intima medial thickness. Circulation. 2002;106:2055-2060.

44. Crouse JR III, Raichlen JS, Riley WA, et al. Effect of rosuvastatin on progression of carotid intima-media thickness in low-risk individuals with subclinical atherosclerosis: the METEOR Trial. JAMA. 2007;297:1344-1353.

45. Nissen SE, Nicholls SJ, Sipahi I, et al. Effect of very high-intensity statin therapy on regression of coronary atherosclerosis: the ASTEROID trial. JAMA. 2006;295:1556-1565.

46. Ballantyne CM, Raichlen JS, Nicholls SJ, et al. Effect of rosuvastatin therapy on coronary artery stenoses assessed by quantitative coronary angiography: a study to evaluate the effect of rosuvastatin on intravascular ultrasound-derived coronary atheroma burden. Circulation. 2008; 117:2458-2466.

47. Brown BG, Zhao XQ, Chait A, et al. Simvastatin and niacin, antioxidant vitamins, or the combination for the prevention of coronary disease. N Engl J Med. 2001;345:1583-1592.

48. Brown G, Albers JJ, Fisher LD, et al. Regression of coronary artery disease as a result of intensive lipid-lowering therapy in men with high levels of apolipoprotein B. N Engl J Med. 1990;323:1289-1298.

49. Brown BG. Maximizing coronary disease risk reduction using nicotinic acid combined with LDL-lowering therapy. Eur Heart J. 2005; 7 Suppl F:F34-F40.

50. Taylor AJ, Sullenberger LE, Lee HJ, Lee JK, Grace KA. Arterial Biology for the Investigation of the Treatment Effects of Reducing Cholesterol (ARBITER) 2: a double-blind, placebo-controlled study of extendedrelease niacin on atherosclerosis progression in secondary prevention patients treated with statins. Circulation. 2004;110:3512-3517.
51. Taylor AJ, Lee HJ, Sullenberger LE. The effect of 24 months of combination statin and extended-release niacin on carotid intimamedia thickness: ARBITER 3. Curr Med Res Opin. 2006;22:2243-2250.

52. Superko HR, King S III. Lipid management to reduce cardiovascular risk: a new strategy is required. Circulation. 2008;117:560-568.

53. McKenney JM, Proctor JD, Harris S, Chinchili VM. A comparison of the efficacy and toxic effects of sustained-vs immediate-release niacin in hypercholesterolemic patients. JAMA. 1994;271:672-677.

54. Zambon A, Gervois P, Pauletto P, et al. Modulation of hepatic inflammatory risk markers of cardiovascular diseases by PPAR-alpha activators: clinical and experimental evidence. Arterioscler Thromb Vasc Biol. 2006;26:977-986.

55. Fruchart JC. Novel peroxisome proliferator activated receptor-alpha agonists. Am J Cardiol. 2007;100(11A):n41-n46.

56. Staels B, Fruchart JC. Therapeutic roles of peroxisome proliferatoractivated receptor agonists. Diabetes. 2005;54:2460-2470.

57. Naik SU, Wang X, Da Silva JS, et al. Pharmacological activation of liver $\mathrm{X}$ receptors promotes reverse cholesterol transport in vivo. Circulation. 2006;113:90-97.

58. Levin N, Bischoff ED, Daige CL, et al. Macrophage liver X receptor is required for antiatherogenic activity of LXR agonists. Arterioscler Thromb Vasc Biol. 2005;25:135-142.

59. Navab M, Anantharamaiah GM, Reddy ST, et al. Oral D-4F causes formation of pre-beta high-density lipoprotein and improves highdensity lipoprotein-mediated cholesterol efflux and reverse cholesterol transport from macrophages in apolipoprotein E-null mice. Circulation. 2004;109:3215-3220.

60. Li X, Chyu KY, Faria Neto JR, et al. Differential effects of apolipoprotein A-I-mimetic peptide on evolving and established atherosclerosis in apolipoprotein E-null mice. Circulation. 2004;110:1701-1705.

61. Navab M, Anantharamaiah GM, Hama S, et al. Oral administration of an Apo A-I mimetic Peptide synthesized from D-amino acids dramatically reduces atherosclerosis in mice independent of plasma cholesterol. Circulation. 2002;105:290-292.

62. Rader DJ. Liver $X$ receptor and farnesoid $X$ receptor as therapeutic targets. Am J Cardiol. 2007;100(11A):n15-n19.

63. Szapary PO, Wolfe ML, Bloedon LT, et al. Guggulipid for the treatment of hypercholesterolemia: a randomized controlled trial. JAMA. 2003;290:765-772.
Vascular Health and Risk Management

\section{Publish your work in this journal}

Vascular Health and Risk Management is an international, peerreviewed journal of therapeutics and risk management, focusing on concise rapid reporting of clinical studies on the processes involved in the maintenance of vascular health; the monitoring, prevention and treatment of vascular disease and its sequelae; and the involvement of

\section{Dovepress}

metabolic disorders, particularly diabetes. This journal is indexed on PubMed Central and MedLine. The manuscript management system is completely online and includes a very quick and fair peer-review system, which is all easy to use. Visit http://www.dovepress.com/ testimonials.php to read real quotes from published authors. 